\title{
Germanica
}

\section{« Ein Unort » - le « non lieu » du souvenir chez Günter Kunert}

" Ein Unort » - der " Unort » der Erinnerung bei Günter Kunert

"Ein Unort"- the "non-place" of the memory in Günther Kunert

\section{Martine Benoit}

\section{(2) OpenEdition}

Journals

Édition électronique

URL : http://journals.openedition.org/germanica/242

DOI : 10.4000/germanica.242

ISSN : 2107-0784

Éditeur

Université de Lille

Édition imprimée

Date de publication : 1 juin 2007

Pagination : $91-101$

ISBN : 2-913857-19-1

ISSN : 0984-2632

\section{Référence électronique}

Martine Benoit, « « Ein Unort » - le «non lieu » du souvenir chez Günter Kunert », Germanica [En ligne] 40 | 2007, mis en ligne le 16 février 2010, consulté le 06 octobre 2020. URL : http://

journals.openedition.org/germanica/242 ; DOI : https://doi.org/10.4000/germanica.242

Ce document a été généré automatiquement le 6 octobre 2020.

(c) Tous droits réservés 


\title{
« Ein Unort » - le « non lieu » du souvenir chez Günter Kunert
}

\author{
" Ein Unort » - der " Unort » der Erinnerung bei Günter Kunert \\ "Ein Unort"- the "non-place" of the memory in Günther Kunert
}

\section{Martine Benoit}

«Eine Handvoll Asche, die auf dieser Erde lastet

unaufhebbar »

(Günter Kunert, Seit dem 42.Jahr des Jahrhunderts)

Comme il est erroné cet adage qui affirme que le temps panse les plaies. C'est tout le contraire. [...] L'angoisse ne se contente pas de revenir, elle occupe d'autres espaces $\mathrm{du}$ quotidien et quand, dans le froid et le vent, je fais le tour de mon jardin, les pieds nus dans mes chaussures, cela ne m'évoque pas le climat d'ici, cela m'évoque la souffrance d'hommes marchant vers la mort. Et il en va ainsi tous les jours ${ }^{1}$.

Le froid d'une journée d'hiver associé à la souffrance de persécutés marchant vers la mort - c'est de ce rapprochement, de cette association, qu'il sera question dans cet article - de cette présence chez Günter Kunert d'un autre lieu, d'un lieu autre au centre duquel il y a le génocide juif et la guerre, le sort des persécutés, le traumatisme de la survivance. On sait l'histoire de Kunert enfant, né d'un couple mixte (Edith Kunert, née Warschauer, est considérée de "race juive » par le régime hitlérien) et déclaré par les Lois de Nuremberg "Mischling ersten Grades », "métis au premier degré », un statut particulièrement exposé 2 . On sait que seule la fidélité d'Adolf Kunert permit à Edith et Günter Kunert de ne pas être déportés pour Theresienstadt et Auschwitz ${ }^{3}$. On sait cette « enfance bouleversée » dont Kunert affirme " qu'on ne peut la quitter, tel l'escargot sa coquille $»^{4}$. Je voudrais travailler ici à partir des nouvelles que Günter Kunert publia entre 1968 et 2006 et qui couvrent ainsi toute la production de l'écrivain ${ }^{5}$, et montrer l'émergence d'un ailleurs intime qui dit le traumatisme de la mémoire, du souvenir indélébile - qui dit la dette vis-à-vis des morts, des disparus qui, à jamais, habitent l'auteur.

2 Il s'agit ici bien sûr, au travers d'une étude de différentes nouvelles, de la reconstruction d'un lieu littéraire, lieu de la remémoration. Dans cette reconstruction, 
je distinguerai trois moments. Je voudrais tout d'abord montrer comment le lieu géographique de référence est, généralement, la ville de Berlin, dont on sait l'importance dans la production, notamment poétique, de Günter Kunert ${ }^{6}$. Je voudrais ensuite analyser les détours littéraires qui rendent possible la constitution de ce lieu autre. On verra finalement que cet ailleurs intime est aussi le lieu de l'angoisse infinie devant un recommencement possible, angoisse perceptible notamment par les nombreuses associations qui convergent toutes vers l'autre lieu que fut Auschwitz, le lieu autre, inconnaissable, de l'anéantissement.

\section{Le contexte géographique}

3 Trois nouvelles permettent de camper «l'endroit » géographique de l'émergence du souvenir. Toutes trois ont Berlin pour décor.

Wie das Leben anfängt ${ }^{7}$, Comment la vie commence, est une nouvelle version du mythe d'Orphée et d'Eurydice. Deux jeunes gens descendent dans les entrailles de Berlin afin de trouver un endroit plus intime; au moment de s'enlacer, ils voient déferler sur eux une mer de rats. Seul le jeune homme arrive à trouver le chemin vers l'air libre, quand il se retourne, il est seul. Telle Eurydice, la jeune fille a disparu dans les ténèbres "Voilà comment la vie commence : par la mort. Par un adieu toujours recommencé "8. On peut avoir une première approche autobiographique de cette nouvelle, penser aux adieux aux proches que Kunert dépeint dans son autobiographie Erwachsenenspiele, penser au grand-père maternel surtout, à cette expérience de la mort toute proche à laquelle Kunert enfant fut confronté. Par-delà cette association, on voit l'importance de ces deux protagonistes, partis dans l'espoir de laisser derrière eux le passé, qui sont rattrapés par celui-ci sous la forme d'un océan de rats. On se rappelle comment la propagande nazie s'était servi d'images animalières pour mieux rabaisser les Juifs, on se rappelle les paroles du film de propagande nazie Jud Süß - les rats, disait le commentateur, "sont sournois, peureux, cruels et apparaissent généralement en bandes. Ils représentent parmi les animaux l'élément de destruction perfide et souterrain. Exactement comme les Juifs parmi les hommes $"{ }^{9}$. Kunert fait des rats les éléments du passé refoulé et suggère que la fuite en avant, le refus du travail sur le passé est une impasse. Le jeune homme quant à lui aura à se confronter à ce passé, le bleu azuré qui domine la fin de la nouvelle suggère d'ailleurs un certain espoir dans ce destin.

Allägliche Geschichte einer Berliner Straße est une nouvelle qui a une place un peu à part dans l'œuvre novellistique de Kunert tant elle accompagne la production de cet auteur depuis sa première édition. Cette nouvelle est en effet parue pour la première fois en

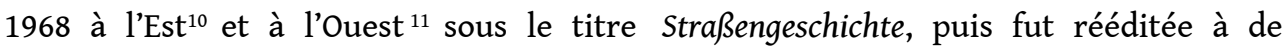
nombreuses reprises, notamment à l'Est en 1979 avec deux autres nouvelles et des gravures de Kunert lui-même sous le titre Drei Berliner Geschichten, ou bien dans Auf Abwegen und andere Verirrungen en 1988, et a été reprise aujourd'hui encore, en 2006, dans le dernier volume de nouvelles paru, Irrtum ausgeschlossen. Dans un bel article de présentation, Patrick Charbonneau relevait la manie chez Kunert de republier certaines nouvelles, notant qu'on pourrait faire à Kunert le reproche de «faire du neuf avec du vieux $»^{12}$. Dans une émission où Kunert, invité, parlait de son dernier recueil de nouvelles, Irrtum ausgeschlossen, le journaliste relevait d'emblée que seules six nouvelles 
étaient inédites ${ }^{13}$. Dans le contexte du présent article, il est intéressant de souligner cette insistance qui dit l'importance de ce texte pour son auteur et qui fait sens.

Dans cette Histoire ordinaire d'une rue de Berlin, il est question d'un Juif allemand contraint à l'exil, David Platzker, qui porte d'ailleurs bien et mal son nom : il est le Juif qui n'a plus sa place (Platz) en Allemagne et qui part avant que ne lui soit imposé le port de l'étoile jaune, « l'étoile de David» justement ${ }^{14}$. Avant de partir, David Platzker prend soin de ramasser sa rue avec ses habitants, de l'enrouler et de glisser ce rouleau sous ses vêtements. Interné pendant son exil, il déroule de temps en temps «sa » rue, fuit ainsi en pensée sa nouvelle condition, se plonge dans la contemplation de ses habitants qui continuent d'aller au travail, la cafetière émaillée à la main, de ses habitantes, à la poitrine toujours accueillante et au sourire inlassablement doux. De retour en Allemagne, le premier mouvement de David Platzker est de remettre la rue à sa place. Mais le lendemain, après une nuit blanche, David Platzker a un mouvement de recul : il veut reprendre «sa » rue, pour continuer à vivre dans «son » Berlin, le Berlin $\mathrm{d}^{\prime}$ ' avant » - mais il est trop tard, il ne reste de « sa » rue qu'une cafetière émaillée dont s'échappe un liquide rouge.

7 On pourrait tout d'abord relever l'arrière-plan autobiographique de cette nouvelle, en rappelant d'emblée que le grand-père maternel auquel Kunert était si attaché portait le prénom de David. De plus, on notera qu'en avril 2005, Kunert accepta de participer à une série d'émissions télévisées retransmises à la radio dans laquelle vingt-cinq artistes racontaient leur expérience de la fin de la guerre. Kunert rapportait alors combien il était difficile et dangereux de trouver de l'eau potable et que lors de ces « expéditions fatales ", il n'était pas rare de se trouver sous les bombardements - et le jeune homme de s'étonner du changement de physionomie des rues: "Les maisons bien sûr changeaient. Il manquait ici et là des bouts de façades, des balcons, une maison tout entière avait soudainement disparu de la rue. C'était très bizarre $»^{15}$.

8 Outre cette dimension biographique, cette nouvelle marque l'impossible réconciliation et l'impossible retour, l'impossibilité de la rencontre entre Juifs et Allemands après la Shoah. Le sang qui coule à la fin suggère le sang d'un monde disparu, le sang des Juifs assassinés qui ne rentreront pas, il suggère l'horreur et le vide. Peu importe finalement la sauvegarde ou pas du décor - l'impossibilité, c'est de retrouver l'intimité de la ville et de ses habitants, la disparition irréparable touche aussi les lieux. On note en outre au détour d'une phrase que le texte place cette "rue de Berlin» à proximité de la Frankfurter Allee : on est dans le fameux "Quartier des Granges », le Scheunenviertel, un quartier pauvre, mal famé, où les Ostjuden chassés par les pogromes de Russie et de Pologne trouvèrent refuge, un des lieux de la vie juive à Berlin avant 1933 - un quartier que Kunert avait présenté dans un essai comme un lieu disparu, anéanti, annihilé, vide de ses habitants - un « Unort », un « non lieu » ${ }^{16}$.

9 L'histoire de Fahrt mit der S-Bahn ${ }^{17}$, Voyage en métro, participe de ce même mouvement d'aspiration à un ailleurs d'avant, d'avant la Catastrophe, d'avant la perte d'un monde et la disparition des proches. Un «Je » aperçoit lors d'un trajet en métro entre deux stations une fenêtre éclairée qui s'ouvre sur une pièce ${ }^{18}$. Dans la pièce entraperçue, se tient ce même "Je ", une pomme à la main, entouré de ses proches et d'un ami, que le «Je » reconnaît bien qu'il en ait oublié l'existence ${ }^{19}$. À la station suivante, le «Je » se précipite pour retourner en sens inverse, revoir à nouveau la scène - entre-temps, le «Je » de la fenêtre a mangé sa pomme - et la vision se fait plus précise : le narrateur reconnaît toutes les personnes présentes, «la plupart avaient disparu depuis, avaient 
été brûlés ou assassinés ou s'étaient exilés ou avaient vieilli $»^{20}$. L'atmosphère qui émane de cette scène est "d'un calme inhabituel comme je ne l'avais jamais vécu ». Puis, « Je » quitte la station à la recherche de cette fenêtre, en vain. Chaque semaine, le narrateur reprend le métro, afin de revoir, encore et toujours, cette même fenêtre et cette même scène. Et l'épilogue dit le désir du narrateur : arriver une fois, une seule, à entrer par la fenêtre, à " s'unir avec soi-même " et ainsi à être " rédimé " ${ }^{21}$, lui et toute la ville de Berlin avec lui - arriver une fois, une seule, à remonter le temps, à retrouver le Berlin d'avant, avec la rue de David Platzker qui y aurait sa place, à retrouver les morts et les disparus. C'est bien ce lieu autre qui se reforme, celui du traumatisme et du souvenir, celui de l'espoir impossible ici, du retour en arrière que seule la poésie, seul l'écrit, peuvent évoquer.

\section{L'émergence du lieu du souvenir}

10 On analysera ici deux détours littéraires qui permettent l'émergence du souvenir, la perception olfactive d'une part, la présence de l'écrit et du livre d'autre part.

11 La nouvelle Auf Abwegen ${ }^{22}$, L'égarement, raconte l'histoire de deux couples de marcheurs qui, perdus dans une forêt, s'arrêtent dans une maison laissée à l'abandon puis repartent - mais un des marcheurs reste en arrière, dans la maison. Il s'agit de Schulz, le meneur de la troupe, qui n'arrive plus à se dégager des odeurs de cette maison : « Ça sent comme à la guerre. Comme après une offensive aérienne ou après des tirs d'artillerie ${ }^{23}$. Ce sont des "odeurs sinistres. Des odeurs meurtrières. Une puanteur annonciatrice de mort mais c'est comme une drogue $»^{24}$. Et Schulz de ne plus bouger alors que les autres repartent, et de crier : « Heimat ! Heimat ! ».

On peut lire cette nouvelle de Kunert comme une version moderne de Hans et Gretel. À l'inverse de Grimm, la maison est déjà détruite à l'arrivée des protagonistes ; à l'inverse de Grimm, le conte ne se termine pas sur une délivrance, Schulz reste enfermé. On connaît la lecture de Bettelheim ${ }^{25}$ : Hans et Gretel montre le difficile chemin que l'enfant doit parcourir pour gagner son autonomie, s'affirmer face au monde. On sait combien la maison est lourde de symboles : elle représente la mère, le corps de la mère qui nourrit l'enfant, la symbiose originelle, qui peut devenir menaçante. Schulz ne peut plus se détacher de cette symbiose, il retourne au stade de la régression qu'il ne veut plus quitter. Mais qui donc s'est égaré ici? Est-ce vraiment Schulz qui, incapable de vivre dans la société d'après-guerre, cherche refuge dans les odeurs de son enfance et de la guerre ? N'est-ce pas plutôt la maison Allemagne qui a suivi le chemin du refoulement et du refus du passé, un chemin moralement peu recommandable? Schulz s'emprisonne dans une pensée dont il ne peut se détacher, il a trouvé une nouvelle Heimat, faite des souvenirs de l'enfance mais aussi des expériences de la guerre. Il ne trouve pas sa place dans la société allemande telle qu'elle s'est construite, dans le déni et le mensonge sur le passé. Il préfère rester en arrière, s'enfermer dans la maison, il ne peut «s'en sortir».

13 La nouvelle Legende vom Schal ${ }^{26}$, Légende d'une écharpe, raconte l'histoire d'un jeune homme dans le Berlin de l'immédiat après-guerre. Hildebert, dit Berti, qui fait du marché noir pour nourrir ses parents et leur offrir le plus beau Noël possible, voit un jour entrer dans l'appartement familial l'ancien propriétaire, E. Löwy, dénoncé à l'époque par le père de Berti engagé dans la SA et qui a survécu à la déportation. En 
repartant, E. Löwy laisse derrière lui l'écharpe qui l'aidait à cacher la cicatrice laissée par un coup de pelle reçu au camp. Berti disparaît sans laisser de trace ${ }^{27}$.

On s'arrêtera sur le nom même du protagoniste, E. Löwy - Juif survivant auquel ne reste du prénom qu'une initiale, dont le prénom a - presque - disparu, le prénom dont on sait l'importance dans la construction de la personnalité et l'affirmation de soi - une initiale qui, cependant, prend tout son sens au fil de la nouvelle par l'évocation du personnage d'Elie. Quant au patronyme Löwy ou Loewy, il fait partie, selon la très brillante étude de Dietz Bering sur la stigmatisation antisémite par le nom, des noms typiquement juifs que le Ministère de la Justice de la République de Weimar reconnaissait comme un des patronymes les plus utilisés par la propagande antisémite à des desseins de raillerie et d'attaque ${ }^{28}$. Le choix même du nom, E. Löwy, dit ainsi à la fois l'exclusion et le désespoir, du prophète Elie ne restant que la seule initiale.

Dans la nouvelle de Kunert, deux événements préparent en quelque sorte la venue d'E. Löwy, au centre desquels se trouve l'écrit. Un vieux livre d'art tout d'abord, que Berti rapporte de ses échappées nocturnes et dans lequel celui-ci trouve une reproduction du tableau de Rembrandt représentant un rabbin. Un rêve ensuite, Berti rêvant cette nuit-là du rabbin de Rembrandt, penché sur un in-folio aux pages vides: «Pas une lettre n'apparait, pas un hiéroglyphe, aucune écriture d'aucune sorte. Tout a disparu. Sans laisser de traces $\aleph^{29}$. À cette disparition du signe répond l'arrivée d'E. Löwy, qui s'arrête dans le salon, auprès du buffet et en sort le verre de Pessah, le verre réservé à Elie le soir de la Pâque juive, Elie dont l'arrivée annonce celle du Messie. Et E. Löwy de s'écrier : «Mais le Messie est fait de cendre. Elie aussi. Et ceux qui ont bu dans le verre. Seul le verre a survécu quoique si fragile - et pourquoi n'est-il pas brisé comme ceux qui l'ont utilisé ? $»^{30}$. À l'inverse d'une relique créée pour témoigner de l'existence avérée du saint chrétien dont elle est censée être un reste et qui par là même atteste réellement de la "vraie religion ", cet objet rituel n'est pas une relique, pas même un objet obsolète; il indique l'anéantissement d'une foi jusqu'à l'anéantissement d'une absence, celle d'Elie. Maintenant le verre seul ne sert plus, personne ne le met plus sur la table de Pessah. Il n'est pas une relique, il peut indifféremment être brisé: l'attente messianique n'a pas été déçue; elle-même a disparu.

E. Löwy repart en oubliant son écharpe, cette écharpe dont rêve Berti depuis tant de jours : «Un vœu s'est réalisé, de manière inattendue. Mais le prix à payer est trop élevé $»^{31}$. Berti s'enfuit de chez lui. Et la nouvelle se clôt sur un épilogue à couper le souffle. À la poste centrale de Buenos Aires, un paquet au nom d'E. Löwy attend. Au bout de quelques années, le paquet est ouvert et il en sort un tissu troué par les mites. Ces lambeaux sont jetés dans un incinérateur et s'échappent en fumée - ultime évocation du génocide, confirmation ultime de l'impossible réconciliation, si bien ressentie et comprise par le tout jeune Berti.

Irrtum ausgeschlossen ${ }^{32}$, L'erreur est exclue, est une nouvelle histoire de livre, d'odeurs de papier, d'odeurs du passé. Un narrateur rapporte l'histoire d'Alwin, son ami, qui dit avoir vu par deux fois chez un bouquiniste son grand-père, mort dix ans auparavant. La première fois, Alwin est en train de feuilleter un livre dans le couloir entre l'entrée de la librairie et une pièce arrière quand son grand-père surgit, portant son habituel paletot et son vieux chapeau. La seconde fois, le vieil homme lui fait même un signe de reconnaissance. Dans cette nouvelle, les références autobiographiques paraissent évidentes, le paletot du vieil homme, son chapeau, son allure évoquent la figure du 
grand-père Warschauer. La magie de cette nouvelle tient dans le fait que plus le récit avance et plus le lecteur est déstabilisé : convaincu d'emblée, comme le narrateur, de l'affabulation d'Alwin, le lecteur voit le doute s'installer. Le lieu du souvenir vient se superposer au présent et troubler les personnages de l'histoire et son lecteur. Passé et présent se juxtaposent, les limites temporelles disparaissent et permettent à cet autre lieu d'émerger dans le présent et de le brouiller.

\section{L'angoisse du recommencement} identifie bien vite à un homme (on pense à K., bien sûr) trouve un matin (on se souvient $\mathrm{du}$ début du Procès) sur la table de son déjeuner un écrit lui intimant de se rendre le 5 novembre de l'année courante à 8 heures du matin dans la cabine 18 des toilettes pour hommes de la gare centrale pour son exécution. L'homme cherche en vain de l'aide auprès de ses amis qui se demandent, après son passage, s'ils ont bien fait de lui ouvrir. Il demande conseil à son avocat qui l'encourage à se rendre à la convocation, parle de faute de frappe - il ne faut pas lire "Hinrichtung " mais "Einrichtung", installation, il faut avoir "confiance $»^{34}$. L'homme se rend au rendez-vous imposé, il prend le chemin de la gare, tel le personnage de cette très brève nouvelle de Kafka, Gib's auf - à la différence fondamentale que, chez Kunert, l'homme trouve son chemin, un chemin qui mène à la mort : au bout d'un quart d'heure, deux hommes viennent retirer le cadavre de la cabine 18 des toilettes (et on se rappelle à nouveau la fin du Procès, décrite de manière si crue). La nouvelle est close par une dernière phrase, en forme d'épilogue : «[...] tout le monde savait que jamais aucun train n'était arrivé ni n'était reparti [de cette gare] bien que planât souvent au-dessus de son toit la fumée de prétendues locomotives $»^{35}$. Et cet épilogue dit tout : entre Kafka et Kunert, entre Gib's auf et Zentralbahnhof, il y a Auschwitz, il y a les chambres à gaz et les crématoires - il y a le traumatisme de la survivance. De nombreux détails mettent en relief le trauma. Le manque de réaction tout d'abord, de l'homme lui-même, des amis, de l'avocat suggérant l'attitude symptomatique de la grande masse sous le Troisième Reich : mieux valait ne pas voir, ne pas savoir ${ }^{36}$. Cette gare ensuite, tellement vide à une heure d'affluence, dont le sol est incessamment nettoyé d'un étrange liquide de désinfection censé dissimuler les crimes et dont les toilettes évoquent les fausses douches $\mathrm{d}^{\prime}$ Auschwitz ${ }^{37}$. Et cette précision dans les chiffres, qui questionne : le 5 novembre si proche d'une autre nuit de novembre, funeste, qui marqua une nouvelle escalade de violence anti-juive en Allemagne. Quant au 18, on rappellera que selon la guématria, cette méthode d'exégèse biblique qui établit une correspondance entre les lettres, les mots et les versets de la Torah d'une part, les nombres d'autre part, le chiffre 18 signifie «vie », le mot «haï» (vie en hébreu) s'écrivant avec les lettres «chait» (huitième lettre de l'alphabet) et "youd» (dixième lettre de l'alphabet) ${ }^{38}$ - détail cruel que cet homme qui entre avec confiance, euphorie même, dans la cabine 18, chiffre d'une croyance violemment démentie par une mise à mort cynique.

L'action de Andromeda zur Unzeit ${ }^{39}$ est située au $\mathrm{xxx}^{\mathrm{e}}$ siècle de l'ère chrétienne, à une époque où la nourriture manque sur terre et où des transferts de population, par fusées 
entières, sont effectués toutes les nuits pour d'autres planètes de la constellation de Cassiopée, des planètes semblables « au jardin d'Eden, loin de toute misère terrestre »40 - le tout est retransmis à la télévision. Pour être candidat au départ, il faut être destinataire d'une carte verte. Dans cette nouvelle, on ne trouve ni narrateur ni personnage, juste un « on » vague et distant. Dans la nuit du mardi 10 avril 3000, une chose étrange arrive : le ciel présenté par la retransmission télévisuelle n'est pas un ciel d'avril, les constellations ne sont pas de saison. Le lendemain, soulagement : ce ciel de novembre montrant la Nébuleuse d'Andromède a disparu. Mais le doute s'est installé, une inquiétude, " comme si une porte s'ouvrait non pas sur une pièce accueillante mais sur un abîme sans lumière, une obscurité inconnue ${ }^{41}$. Pour se soulager un peu, « on " en parle autour de soi, à ses amis, ses connaissances. Et trois jours après arrive la carte verte annonçant le départ imminent.

Les associations ici sont nombreuses. La colonne d'hommes et de femmes traînant leur bagage avant de monter dans une immense fusée rappelle d'autres colonnes menant vers une mort atroce et dont les dépouilles finirent en fumée dans les lugubres cheminées des fours. Ce ciel de novembre en plein mois d'avril suggère un lien entre le boycott d'avril 1933 et les pogromes de novembre 1938. Il y a aussi cette étrange coïncidence entre la visite aux amis et la réception de la carte verte, qui suppose une dénonciation ou une participation plus active encore. Et la destination aussi est étrange, la constellation de Cassiopée, dans l'hémisphère boréal, rappelant cette autre attirance pour la pureté nordique, rappelant cette autre folie de l'aryanité. Tout ici respire le faux-semblant, le camouflage de la mort, et évoque le secret autour de ce que les nazis nommèrent, par un euphémisme, « Solution finale ».

\section{Conclusion}

On voit assez comment les nouvelles présentées ici nous mènent toutes à ce lieu autre de la mémoire traumatique, à ce lieu où le traumatisme se lie à l'angoisse pour suggérer l'horreur de ce que Paul Celan préférait ne pas nommer en disant «Das was geschah »" On ne s'étonnera pas d'apprendre que Günter Kunert, prié par l'hebdomadaire Die Zeit de présenter son « livre du siècle », choisit justement Si c'est un homme de Primo Levi. On sait ce sentiment chez Günter Kunert, que seul le hasard a permis qu'il survive, que seul le hasard a empêché que les chaussures du jeune garçon qu'il était alors ne viennent grossir ce tas qui, aujourd'hui encore, bouleverse les visiteurs d'Auschwitz. Les nouvelles de Kunert montrent la présence de ces images de mort et d'assassinat dans l'univers intime de l'auteur, elles disent le passé tragique et montrent le lieu de l'absence et de la disparition irréversibles. Elles laissent émerger ce «Unort » du souvenir terrible et angoissant. 


\section{NOTES}

1. Günter Kunert: Die Botschaft des Hotelzimmers an den Gast - Aufzeichnungen (éd. Hubert Witt), München/ Wien, Hanser, 2004, p. 95-96.

2. Voir à ce sujet Martine Benoit: «“...et c'est devenu mon destin” - Günter Kunert et la judéité », p. 73-90, in : Lectures d'une œuvre - Günter Kunert, ouvrage collectif coordonné par MarieHélène Quéval, Paris, Éditions du Temps, 2000.

3. On pourra lire à ce sujet le texte que Kunert consacra à son père «Ohne Bilanz " paru dans Günter Kunert, Ortsangaben, Berlin, Aufbau, 1970.

4. Günter Kunert : Irrtum ausgeschlossen, München, Hanser, 2006, p. 8.

5. Poète, essayiste, romancier, dessinateur, Kunert est aussi un écrivain de nouvelles, ce que Manfred Durzak, dans une interview donnée en 1977, saluait avec force: "Man darf ohne Übertreibung sagen, dass Ihr Name und die Geltung der aktuellen deutschen Kurzgeschichte, in der DDR und weit darüber hinaus, fast synonym sind» (Manfred Durzak: Die deutsche Kurzgeschichte der Gegenwart - Autorenporträts - Werkstattgespräche - Interpretationen, Stuttgart, Reclam, 1980, p. 84).

6. Voir Martine Benoit : " "Fremd daheim", étranger chez soi - le sentiment d'exil chez Günter Kunert », Tsafon-Revue d'études juives du Nord 49, printemps-été 2005, p. 85-98.

7. Wie das Leben anfängt a été publiée pour la première fois dans Die Beerdigung findet in aller Stille statt (München, Hanser, 1968) et rééditée dans le recueil Auf Abwegen und andere Verirrungen (München, Hanser, 1988, p. 12-19 - les citations du texte se réfèrent à cette édition).

8. Günter Kunert, Wie das Leben anfängt, op. cit., p.19.

9. Cité d'après Anne Grynberg: La Shoah - L'impossible oubli, Paris, Découvertes Gallimard, 1995, p. 35. On rappellera que le gouvernement de Vichy usa et abusa lui aussi de l'image des rats dans sa propagande antisémite.

10. Dans le recueil Kramen in Fächern - Geschichten. Parabeln. Merkmale, Berlin, Aufbau-Verlag, 1968.

11. Dans le recueil Die Beerdigung findet in aller Stille statt, op. cit.On attend ici avec impatience la publication par Nicolai Riedel de la Internationale Günter-Kunert-Bibliographie annoncée pour fin 2007.

12. Patrick Charbonneau: "Les grands thèmes de Günter Kunert - À propos du recueil Auf Abwegen und andere Verirrungen ", Allemagne d'aujourd'hui, janvier-mars 1990, p. 138-143.

13. Michael Opitz: «Günter Kunert erzählt von Irrtümern », émission du 16 août 2006, décryptée à l'adresse : www.dradio.de/dlf/sendungen/buechermarkt/.

14. C'est une ordonnance du 21 septembre 1941 qui impose le port de l'étoile jaune en Allemagne pour toute personne âgée de plus de six ans et considérée comme juive. On notera que Günter Kunert, avec son statut de métis au père aryen et à la mère juive, ne porta pas l'étoile jaune.

15. Décryptage de l'émission à l'adresse http://www.wdr.de/tv/wdr-dok/archiv/2005.

16. Günter Kunert : «Ein Unort», p. 7-9, in : Eike Geisel : Im Scheunenviertel - Bilder, Texte und Dokumente, Berlin, Severin und Siedler, 1981.

17. Fahrt mit der S-Bahn est parue pour la première fois en 1968 dans le recueil Kramen in Fächern, puis fut reprise dans Auf Abwegen und andere Verirrungen (les citations font référence à cette édition).

18. On peut penser que là aussi, un souvenir d'enfance a préludé à l'écriture de cette nouvelle. Dans son introduction au livre que consacra Eike Geisel au Scheunenviertel de Berlin en 1981, Kunert parle des sœurs de son grand-père maternel qui habitaient dans la Keibelstraße, une rue qui donnait sur l'Alexanderplatz, de leur immeuble dans lequel il venait souvent enfant. Une des fenêtres de l'appartement donnait sur un mur « qu'un homme aurait pu toucher en tendant le 
bras » (Kunert : «Ein Unort», op. cit.). Ce rapprochement permet de supposer que nous restons, dans l'esprit de l'auteur, dans le Scheunenviertel.

19. On peut penser ici au portrait du jeune Waldemar que Kunert brosse dans son autobiographie, ce jeune garçon que Kunert aimait tant parce qu'il n'avait fait aucune remarque lorsqu'il aperçut, un jour, cousue sur le manteau du grand-père Warschauer, l'étoile de l'exclusion - Waldemar qui mourut dans un foyer en Bohême et que Kunert attendit longtemps encore après la guerre (cf. Kunert : Erwachsenenspiele, op. cit., p. 33).

20. Günter Kunert, Fahrt mit der S-Bahn, op. cit., p.49.

21. Ibid., p.50.

22. Auf Abwegen est une nouvelle inédite du recueil Auf Abwegen und andere Verirrungen, München, Hanser, 1988.

23. Günter Kunert, Auf Abwegen, op. cit., p.297.

24. Ibid., p.297.

25. Voir Bruno Bettelheim, Psychanalyse des contes de fées, traduit de l'américain par Théo Carlier. Paris : Hachette, 1976 (Pluriel), notamment le développement sur " Jeannot et Margot ».

26. Legende vom Schal est parue pour la première fois en 1968 dans le recueil Kramen in Fächern, puis fut reprise dans Auf Abwegen und andere Verirrungen (les citations se réfèrent à cette édition).

27. On pourra se reporter à un souvenir que Kunert évoque dans son autobiographie, Erwachsenenspiele (p.97-98), celui de son seul cousin revenu de déportation et de leur brève rencontre en 1945.

28. Dietz Bering: Der Name als Stigma - Antisemitismus im deutschen Alltag 1812-1933, Stuttgart, Klett, 1987, notamment p. 222.

29. Günter Kunert, Legende vom Schal, op. cit., p. 26.

30. Ibid., p. 30.

31. Ibid., p. 31.

32. Nouvelle inédite du recueil éponyme paru en 2006.

33. Zentralbahnhof est parue pour la première fois en 1968 dans Kramen in Fächern, op. cit.; elle est reproduite dans Jochen Richter (éd.) : Günter Kunert - Schatten entziffern, Leipzig, Reclam, 1995 (les citations font référence à cette édition).

34. Günter Kunert, Zentralbahnhof, op. cit., p.197.

35. Ibid., p. 198.

36. Voir Manfred Durzak : Die deutsche Kurzgeschichte der Gegenwart, op. cit., p. 344.

37. Voir l'interview de Günter Kunert dans Manfred Durzak, ibid., p. 101 : «Obwohl sich die Geschichte bemüht, dauernd Assoziationen hervorzurufen. Assoziationen an real vorhandene Tötungsmechanismen. Also z. B. diese Toilette: Das ist eigentlich schon fast so wie die Pseudobäder in den Vernichtungslagern. Da sind ja Ähnlichkeiten anvisiert ».

38. Un grand merci à Martine Hamiache pour ses explications éclairantes.

39. Andromeda zur Unzeit est parue en 1968 dans le recueil Die Beerdigung findet in aller Stille statt et fut rééditée en 1988 dans Auf Abwegen und andere Verirrungen (les citations se réfèrent à cette édition).

40. Günter Kunert, Andromeda zur Unzeit, op. cit., p.267.

41. Ibid., p. 268.

42. Voir le « Discours de Brême » en 1958. 


\section{RÉSUMÉS}

À partir de l'analyse de nouvelles que Günter Kunert publia sur quelque quatre décennies, cet article propose de reconstruire le lieu du souvenir et de la remémoration propre à cet auteur. On verra ainsi émerger un ailleurs intime qui dit le traumatisme du souvenir indélébile mais aussi, encore et toujours, l'angoisse du recommencement possible, une angoisse perceptible notamment dans les nombreuses associations qui, toutes, convergent vers l'autre lieu que fut Auschwitz, le lieu autre, inconnaissable, de l'anéantissement.

Dieser Artikel nimmt sich Kurzgeschichten vor, die Günter Kunert im Laufe von knapp vierzig Jahren verfasste und publizierte. Es geht hier um die Suche nach einer literarischen Rekonstruktion des inneren " Unortes » der Erinnerung - eines « Unortes », der von einem tiefen Trauma erzählt, aber auch von der Angst, dass das, was Auschwitz ermöglichte, das, was nach Auschwitz führte, wieder anfangen könne.

From the analysis of short stories that Günter Kunert published over around four decades, this article offers to rebuild the place of memory and recall that are proper to this author. We will thus see emerging an inner elsewhere that tells the trauma of the indelible memory but will also see, again and again, the anxiety of the possible resumption, a anxiety discernible namely in the numerous associations that all converge to the other place that Auschwitz were; the other place, unknown of the annihilation.

\section{INDEX}

Mots-clés : Shoah, mémoire, souvenir

oeuvrecitee Wie das Leben anfängt, Straßengeschichte, Drei Berliner Geschichten, Auf Abwegen und andere Verirrungen, Irrtum ausgeschlossen, Die Beerdigung findet in aller Stille statt, Alltägliche Geschichte einer Berliner Straße, Erwachsenenspiele

\section{AUTEURS}

\section{MARTINE BENOIT}

Université Charles-de-Gaulle - Lille 3 